\section{Perspektiven der weiteren Projektarbeit}

\author{
von Armin Grunwald, Forschungszentrum \\ Karlsruhe
}

Das HGF-Projekt „Global Zukunftsfähige Entwicklung - Perspektiven für Deutschland" übersetzt als problemorientierte Forschung die gesellschaftliche Wahrnehmung, dass gegenwärtige Wirtschaftsformen in einigen Aspekten massive Defizite in Bezug auf Zukunftsfähigkeit zur Folge haben, in eine wissenschaftliche Agenda, die sich mit Operationalisierungsmöglichkeiten und -problemen des Nachhaltigkeitsprinzips für Deutschland befasst. Übergeordnetes Ziel ist, die wissenschaftliche Diskussion über Nachhaltigkeit voranzubringen und Orientierungs- und Handlungswissen für die gesellschaftlichen Akteure zu erarbeiten, die bei der Realisierung einer nachhaltigen Entwicklung in Deutschland mitwirken. Die HGF kommt damit einerseits ihrer Verpflichtung zur Vorsorgeforschung im nationalen Interesse nach. Andererseits können durch dieses Projekt vielfältige Kompetenzen von HGF-Einrichtungen themenzentriert zusammengeführt werden. Das Projekt wird im Jahr 2002 abgeschlossen.

Mit der Zielsetzung, Orientierungs- und Handlungswissen für die im Nachhaltigkeitskontext relevanten gesellschaftlichen Akteure bereitzustellen, begibt sich das HGF-Verbundprojekt in einen Bereich, der hauptsächlich durch außerwissenschaftliche Problemwahrnehmungen, normativ kontroverse Voreinstellungen und konfligierende Interessen geprägt ist. In dieser Situation - die typisch für problemorientierte Forschung ist -, ergeben sich besondere Anforderung an die Formulierung wissenschaftlicher Aufgabenstellungen, soll nicht Wissenschaft auf die Rolle eines Interessenvertreters für bestimmte Positionen reduziert werden. Im vorliegenden Fall bedeutet dies, dass die Übersetzung des normativen Nachhaltigkeitskonzeptes in ein wissenschaftliches Forschungsprogramm nicht „missionarisch" sein darf. Sie kann die in Gerechtigkeitsidealen enthaltene Normativität nicht selbst als gültig setzen, sondern muss sich kon- ditional darauf beziehen: „Wenn man das Gerechtigkeitspostulat als normativen Ausgangspunkt verwendet, hat dies folgende Implikationen: ....... Über die Berechtigung oder Inkraftsetzung des Postulates selbst bzw. seiner Konkretisierungen auf der Ebene der Nachhaltigkeitsziele kann nicht wissenschaftlich entschieden werden; dies ist Sache der Gesellschaft in ihren dafür legitimierten Verfahren und Institutionen. Die normative Ebene kann jedoch theoretisch-systematisch aufgearbeitet werden, um sie transparent und argumentationszugänglich zu machen.

Nachhaltigkeit muss weiterhin, dazu verpflichtet die zugrundeliegende Gerechtigkeitsproblematik, in globaler Perspektive analysiert werden. Gehandelt werden kann allerdings oft nur oder besser im lokalen, regionalen oder nationalen Rahmen. Die Herstellung des Bezuges zwischen einer global nachhaltigen Entwicklung und ihren Anforderungen und Realisierungsbedingungen einerseits und den Möglichkeiten und Grenzen nationaler bzw. regionaler Steuerungsmaßnahmen andererseits wird daher eine wesentliche Aufgabe des Projektes sein. Beiträge zur Konkretisierung des Leitbilds einer nachhaltigen Entwicklung für Deutschland sollen durch die Entwicklung und Analyse von Handlungsoptionen und Strategien auf nationaler Ebene sowie für einzelne Aktivitätsfelder (z. B. Bauen und Wohnen, Mobilität, Landwirtschaft und Ernährung) geleistet werden. Ein Fokus des Vorhabens besteht darin, die verschiedenen durch technologische und gesellschaftliche Innovationen erreichbaren Effizienz- und Konsistenzsteigerungspotentiale zur Erreichung von Nachhaltigkeitszielen zu untersuchen. Diese werden auf ihre Effektivität, Folgewirkungen und Realisierungsbedingungen hin analysiert und bewertet.

\section{Konzeptioneller Ansatz}

Da mit dem Leitbild Nachhaltigkeit die Vorstellung verbunden wird, Verbesserungen der ökonomischen und sozialen Lebensbedingungen mit der langfristigen Sicherung der natürlichen Lebensgrundlagen in Einklang zu bringen, ist das integrative Konzept ein theoretischkonzeptionelles Kernstück des Vorhabens (vgl. den Beitrag von Jörissen, Brandl, Kopfmüller, Paetau in diesem Schwerpunkt). Das normati- 
ve Postulat inter- und intragenerationeller Gerechtigkeit wird in Form von Regeln operationalisiert, die erstens orientierend im Hinblick auf die Suche nach nachhaltigkeitsrelevanten Entwicklungen, Trends oder Technologien wirken, und die zweitens in die Bewertung von gegenwärtigen Zuständen, Szenarien oder Handlungsoptionen eingehen. Diese Regeln stellen eine „Entfaltung“ der normativen Zielsetzung dar, verbunden mit einer Übersetzung auf bestimmte gesellschaftliche Dimensionen.

Die Ausgangshypothese des Projektes ist, dass durch eine Beachtung des Regelsystems durch die gesellschaftlichen Akteure bzw. Teilsysteme den Anforderungen einer Nachhaltigen Entwicklung entsprochen werden kann (bzw. besser entsprochen werden kann als bei Nichtbeachtung). Diese Hypothese wird im Laufe des Projektes geprüft, wobei insbesondere auf Zielkonflikte zwischen Regeln geachtet wird. Diese Prüfung kann zu Veränderungen, Ergänzungen oder Streichungen im Regelsystem führen. Das integrative Konzept wird auf diese Weise daraufhin überprüft, inwieweit es einerseits als Detektor für Konfliktfelder geeignet ist und andererseits Wege zum konstruktiven Umgang mit diesen Konflikten weisen kann.

Die weitere Konkretisierung der Regeln findet auf der Ebene der Indikatoren statt. Einerseits werden durch theoretische ,topdown"-Überlegungen den Nachhaltigkeitsregeln mögliche Indikatoren zugeordnet, um die Einhaltung der Regeln in geeigneter Form „messbar“ zu machen. Andererseits werden vorhandene Indikatorensysteme im Hinblick auf das Regelsystem gesichtet (vgl. den Beitrag von Coenen in diesem Schwerpunkt) und es werden auf diesem Weg potentielle Indikatoren „bottom-up“ vorgeschlagen. Die Festlegung auf die im Projekt zu untersuchenden Indikatoren erfolgt dann durch einen Vergleich der „top-down“ und der „bottom-up“ gewonnenen Indikatorensysteme. Dieser Abgleich dient einerseits der Konsistenzprüfung, andererseits werden Lerneffekte für den Bezug der Nachhaltigkeitsregeln auf die Aktivitätsfelder erwartet.

Es ist im Rahmen des Projektes weder möglich noch sinnvoll, sämtliche Nachhaltigkeitsregeln in gleich detaillierter Weise zu operationalisieren und diese dann ,flächendeckend" auf die Gesellschaft zu beziehen. Statt- dessen werden Relevanzkriterien herangezogen, um eine begründete Auswahl treffen zu können und an dieser Auswahl die Arbeitsweise des integrativen Ansatzes zu demonstrieren. Die Kriterien für die Auswahl von Regelsätzen und Nachhaltigkeitszielen, die für eine vertiefte Operationalisierung des integrativen Ansatzes herangezogen werden (Komplexitätsreduktion), orientieren sich an der Relevanz für die Situation in Deutschland, an der Konfliktträchtigkeit (Konflikte zwischen dem Nachhaltigkeitsprinzip und gegenwärtigen Trends, Zielkonflikte zwischen Regeln aus verschiedenen Bereichen, Nutzungskonflikte etc.) und an dem Ausmaß vermuteter bzw. erkennbarer Nachhaltigkeitsdefizite und daraus resultierender dringender Handlungsbedarfe.

Die Herstellung des Bezuges zwischen dem integrativen Ansatz und der Ebene empirischer Untersuchungen erfolgt durch den Aktivitätsfelder-Ansatz (vgl. den Beitrag von Klann, Nitsch in diesem Schwerpunkt). Ein wesentlicher Vorteil dieses Ansatzes besteht darin, die Nachhaltigkeitsthematik und vor allem die Auswirkungen einerseits nichtnachhaltiger Wirtschaftsformen, andererseits der Umsteuerung auf Nachhaltigkeit hin, direkt an die Lebenswelt der Betroffenen anbinden zu können (Mobilität, Wohnen, Ernährung etc.). Die Implikationen einer Nachhaltigkeitspolitik für die konkreten Lebensverhältnisse können auf diese Weise besser verdeutlicht werden als andere (lebensweltfernere) Klassifizierungen gesellschaftlicher Bereiche.

Diskussionsprozesse mit Experten und gesellschaftlichen Akteuren werden an verschiedenen Stellen im Projektablauf angesetzt, um einerseits weiteres Experten- und Akteurswissen in das Projekt zu integrieren. So werden in der Analyse und Bewertung der Potentiale von Schlüsseltechnologien Panels einberufen, bestehend aus Wissenschafts- und Technikexperten. Andererseits werden aktivitätsfelderspezifische gesellschaftliche Akteure einbezogen, wenn es um die Aufstellung von Nachhaltigkeitszielen und die Bewertung von Handlungsstrategien in den Aktivitätsfeldern geht.

\section{Arbeitsschwerpunkte}

Es werden die gesellschaftlichen Aktivitätsfelder Mobilität, Bauen und Wohnen, Landwirt- 
schaft und Ernährung sowie Information und Kommunikation einer detaillierteren Analyse unterzogen (inwieweit das Aktivitätsfeld Freizeit und Tourismus hinzugenommen werden kann, wird noch geprüft). Für diese Aktivitätsfelder werden auf nationaler Ebene (unter Berücksichtigung der internationalen Verflechtung)

- bestehende und absehbare Nachhaltigkeitsdefizite und Zielkonflikte analysiert,

- Ziel- bzw. Richtungsvorgaben anhand geeigneter Indikatoren formuliert,

- $\quad$ spezifische Effizienz-, Konsistenz- und Suffizienzpotentiale analysiert und in Szenarien abgebildet,

- Maßnahmen und Instrumente auf ihre Wirksamkeit für die Erreichung der Ziele und auf ihre Implementationsbedingungen hin untersucht,

- aktivitätsfeldspezifische Teilstrategien entwickelt und in ihren Wechselwirkungen zu anderen Aktivitätsfeldern analysiert.

Die Spezifika (Möglichkeiten, Grenzen, Erfordernisse, Nutzungskonflikte usw.) bei der Umsetzung des integrativen Konzepts nachhaltiger Entwicklung auf regionaler Ebene werden in eigenen Fallstudien untersucht. Diese Problematik wird a) in einer regionalen Fallstudie "Ballungsräume" am Beispiel des Großraums Berlin analysiert und b) am Beispiel einer nicht-nachhaltigen Landnutzung in ländlichen Räumen verdeutlicht. Die Möglichkeit einer regionalen Analyse „Küstenregion“ wird noch geprüft.

Hervorgehoben aus den quer dazu angesiedelten Untersuchungsgegenständen sei die Abschätzung der Potentiale schon vorhandener sowie zukünftiger aktivitätsfelder-übergreifender Schlüsseltechnologien zur Erreichung der Ziele einer nachhaltigen Entwicklung sowie zu den Folgewirkungen ihres Einsatzes. Einer vertiefenden Betrachtung werden insbesondere regenerative Energietechnologien, Bio- und Gentechnologien, Informations- und Kommunikationstechnologien, Mikro- und Nanotechnologien sowie neue Materialien unterzogen.

\section{Erwartete Ergebnisse}

Auf diese Weise soll die Nachhaltigkeitsdiskussion in mehrfacher Weise vorangebracht werden. Einerseits geht es um die Belebung der wissenschaftlichen Auseinandersetzung mit diesem Thema; hier soll das Projekt durch den konzeptionellen Ansatz und die Methodik neue Wege für die wissenschaftliche Bearbeitung eröffnen. Andererseits werden relevante gesellschaftliche Akteure angesprochen, wenn sowohl Handlungs- und Orientierungswissen bereitgestellt wird als auch die Umsetzungsbedingungen analysiert werden. So dürfte die Diskussion um das Für und Wider eines nationalen Nachhaltigkeitsplanes, aber auch um eine eventuelle konkrete Ausgestaltung durch das Projekt neue Impulse erhalten. Die Ergebnisse des Projektes werden konkret bestehen in:

- einer kritischen Sichtung und Weiterentwicklung vorhandener Ziel- und Indikatorensysteme und die transparente Herstellung ihrer Bezüge zum Nachhaltigkeitskonzept;

- einer flächendeckenden quantitativen Erfassung nachhaltigkeitsrelevanter Trends, strukturiert nach Aktivitätsfeldern;

- einer vertieften Bestandsaufnahme und Bewertung nachhaltigkeitsrelevanter Entwicklungen in ausgewählten Aktivitätsfeldern unter besonderer Berücksichtigung von Zielkonflikten,

- einer Ausarbeitung von Szenarien und Strategien nachhaltiger Entwicklung in ausgewählten Aktivitätsfeldern für nationale und regionale Belange und der Markierung des politischen Entscheidungsbedarfs und der Entscheidungsspielräume;

- der Analyse von Wechselwirkungen zwischen den Aktivitätsfeldern auf nationaler Ebene und einer Konkretisierung von Nachhaltigkeit in regionalen Fallanalysen,

- einer Analyse und Bewertung der Potentiale von Schlüsseltechnologien im Hinblick auf Nachhaltigkeit,

- der Entwicklung und Weiterentwicklung von Modellen und Instrumenten und dem Aufbau innovativer Software-Tools, z.B. für die Mensch-Modell-Maschine-Simulation (M3 Simulation). 
Die Vision ist, dass auf diese Weise - über die Erreichung der o.g. Ziele hinaus - ein Operationalisierungsinstrumentarium für Nachhaltigkeit entwickelt und erprobt werden kann, das zukünftig für vielfältige Anwendungen weiterverwendet werden kann. Denn das Thema „Nachhaltigkeit" wird auf der gesellschaftlichen und wissenschaftlichen Agenda bleiben. Der Nachhaltigkeitsdiskurs als ein permanenter gesellschaftlicher, politischer, wissenschaftlicher und öffentlicher Diskurs über Natur, Umwelt und die Zukunft der Gesellschaft wird durch das HGF-Projekt weitere Impulse erhalten und vielfältige Lerneffekte ermöglichen.

\section{Kontakt}

Prof. Dr. Armin Grunwald

Forschungszentrum Karlsruhe $\mathrm{GmbH}$

Institut für Technikfolgenabschätzung

und Systemanalyse (ITAS)

Postfach 3640, D-76021 Karlsruhe

Tel.: + 49 (0) $7247-822500$

Fax: + 49 (0) $7247-824806$

E-Mail: Grunwald@itas.fzk.de

$\ll 》$

Schlussbericht zum HGF-Projekt „Untersuchung zu einem integrativen Konzept nachhaltiger Entwicklung: Bestandsaufnahme, Problemanalyse, Weiterentwicklung“"

Coenen, R., Paschen, H., „Überblick über den Abschlussbericht“. März 2000 (9. S.)

Jörissen, J., Kneer, G., Rink, D., Paskalewa, K.: „Synopse zur Umsetzung des Leitbildes der Nachhaltigkeit in konzeptionellen Studien und nationalen Plänen“. Band 1, Dezember 1999, 212 S.

Gray, P.: „Umsetzung des Leitbildes auf unternehmerischer, regionaler und kommunaler Ebene". Band 2, $40 \mathrm{~S}$.

Stransfeld, R.: „Regionale Ökonomie als räumlicher Orientierungsansatz für integrierte Nachhaltigkeit - Eine Bestandsaufnahme“. Materialienband 2.A, März 1999, 46 S.

Stransfeld, R.: „Regionale Ökonomie für integrierte Nachhaltigkeit: Ansatz und Realität“. Materialienband 2.B, März 1999, 47 S.

Fischer, W.: „Zukunftsfähige Entwicklung in den deutschen Biosphärenreservaten“. Materialienband 2.C, April 1999, 16 S.

Dippoldsmann, P.: „Umsetzungen des Leitbildes Sustainable Development auf lokal-gesellschaftlicher Ebene“. Materialienband 2.D, Dezember 1999, 228 S.

Gray, $P$.: „Umsetzungen des Leitbildes einer nachhaltigen Entwicklung in deutschen Unternehmen“. Materialienband 2.E, Dezember 1999, 71 S.

Krumm, R.: „Der Lokale Agenda-21 Prozess in Deutschland: einige wirtschaftswissenschaftliche Anmerkungen“. Materialienband 2.F, März 1999, 15. S.

Jörissen, J., Kopfmüller, J., Brandl, V., Paetau, M.: „Ein integratives Konzept nachhaltiger Entwicklung“. Band 3, Dezember 1999, 202 S.

Coenen, R., Backhaus, R., Schade, B., Weiers, St., Mühle, H.: „Konzeptionelle Aspekte der Entwicklung von Nachhaltigkeitsindikatoren“. Band 4, Dezember 1999, 51 S.

Klann, U., Nitsch, J.: „Verursacherbezogene, konsistente Erfassung von Belastungsbeiträgen und Integration in ein gesamtwirtschaftliches Modell“. Band 5, Dezember 1999, $92 \mathrm{~S}$.

Sydow, A., Rufeger, W., Rose, H., Aßelmeyer, T.: „Bestandsaufnahme zu Potentialen und Grenzen vorhandener Modelle“. Band 6, Dezember 1999, 62 S.

Die Bände sind kostenlos erhältlich bei: Forschungszentrum Karlsruhe, Institut für Technikfolgenabschätzung und Systemanalyse (ITAS), Postfach 3640, D-76021 Karlsruhe, Frau M. Kullmann, Tel.: + 49 (0) 7247 / 825346, Fax: + 49 (0) 7247 / 824806, E-Mail: Kullmann@itas.fzk.de 